\title{
Linear behaviour of the timepix detector for electron diffraction studies
}

\author{
Viktor Emanuel Gillis Bengtsson ${ }^{1}$, Hongyi Xu' ${ }^{1}$, Xiaodong Zou ${ }^{1}$ \\ ${ }^{1}$ Department Of Materials And Environmental Chemistry, Stockholm University, Stockholm, Sweden \\ E-mail: viktor.bengtsson@mmk.su.se
}

The Timepix detector is a hybrid electron detector with counting capability dedicated for recording electron diffraction patterns. The detector has ultra-fast read-out ( $8.3 \mathrm{~ms}$, up to $120 \mathrm{frames} / \mathrm{s})$, high sensitivity and theoretically zero noise. These are especially useful for collecting 3D electron diffraction data from beam sensitive materials for structural analysis, with methods such as Rotation Electron Diffraction (RED), Automated Diffraction Tomography (ADT) and continuous rotation being developed. In recent years many complex unknown structures were solved using 3D electron diffraction methods. Such structures include zeolites, metal-organic frameworks (MOFs), organic compounds and open-framework germanates. The mentioned 3D electron diffraction methods all involve reconstructing the 3D reciprocal space from a number of diffraction patterns taken at different angles. This requires accurate intensities to be recorded for both strong and weak Bragg spots to give an accurate reconstruction, and the exposure time for each image must be low. In order to collect meaningful electron diffraction data, each pixel of the detector has to behave linearly up to its counting limit (11800) against the exposure time and the number of incident electrons. Thus, we tested the behaviour of a Timepix detector installed on a JEOL 2100 TEM with LaB6 filament. We compared the same set of strong, medium and weak Bragg spots in diffraction patterns collected using exposure time ranging from $1 \mathrm{~ms}$ to $262144 \mathrm{~ms}$ with a step size of $2 \mathrm{n}$ ms (where $\mathrm{n}$ equals to $1,2,3 \ldots)$. It was found that the measured intensities of the Bragg spot are linearly proportional to the exposure time between 25 and 11500 counts per pixel. Furthermore, the slopes of the linear region of the measured intensities from the strong, medium and weak Bragg spots against the exposure time were very similar. These results indicate the linear behaviour of the Timepix detector against both the exposure time and the number of incident electron within the dynamical range of the detector. Therefore, electron diffraction patterns collected by the Timepix detector can be quantitatively used for structural analysis by electron crystallography.

[1] Wan, W. et al. (2013). J Appl Crystallogr. 46, 1863-1873.

[2] Yun, Y. et al. (2015). Crysteng. 2, 267-282.

[3] van Genderen, E. et al. (2016). Acta Cryst. A72, 236-242.

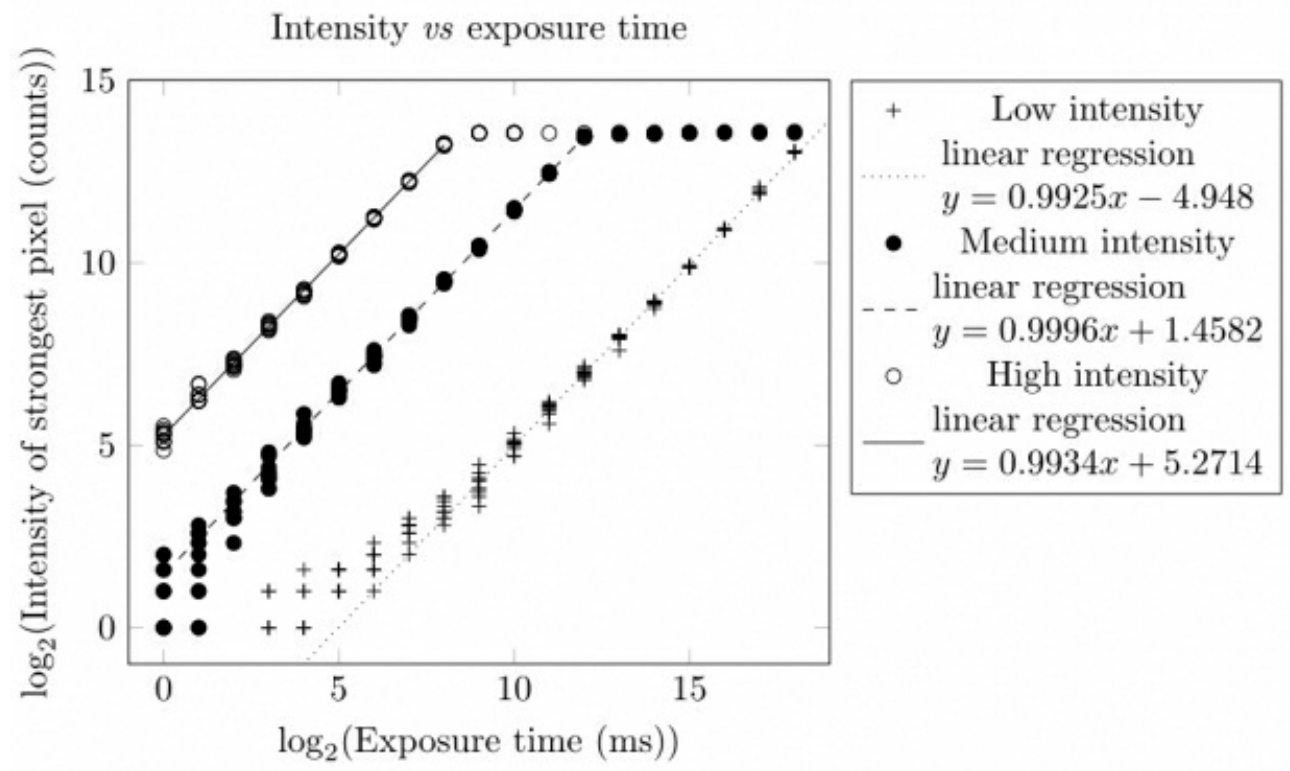

Keywords: Timepix, electron diffraction 